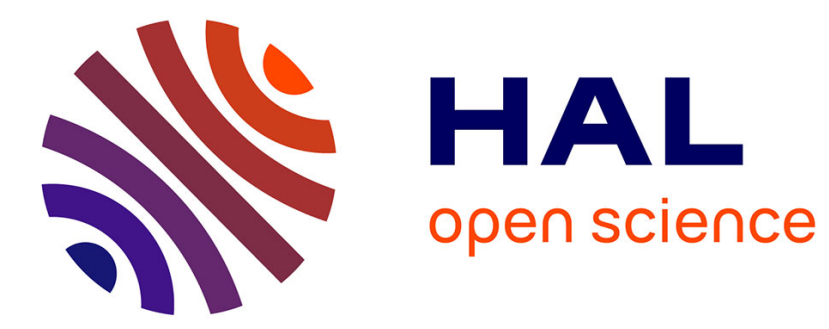

\title{
UN NOUVEAU MODĖLE POUR LE CALCUL DU CHAMP DE PRESSION PARAMÉTRIQUE
}

\author{
F. Coulouvrat
}

\section{To cite this version:}

F. Coulouvrat. UN NOUVEAU MODĖLE POUR LE CALCUL DU CHAMP DE PRESSION PARAMÉTRIQUE. Journal de Physique IV Proceedings, 1992, 02 (C1), pp.C1-649-C1-652. 10.1051/jp4:19921140 . jpa-00251098

\section{HAL Id: jpa-00251098 https://hal.science/jpa-00251098}

Submitted on 1 Jan 1992

HAL is a multi-disciplinary open access archive for the deposit and dissemination of scientific research documents, whether they are published or not. The documents may come from teaching and research institutions in France or abroad, or from public or private research centers.
L'archive ouverte pluridisciplinaire HAL, est destinée au dépôt et à la diffusion de documents scientifiques de niveau recherche, publiés ou non, émanant des établissements d'enseignement et de recherche français ou étrangers, des laboratoires publics ou privés. 


\title{
UN NOUVEAU MODELE POUR LE CALCUL DU CHAMP DE PRESSION PARAMETRIQUE
}

\author{
F. COULOUVRAT \\ Laboratoire de Modélisation en Mécanique associé au C.N.R.S., Université Paris 6, Boîte 162, \\ 4 place Jussieu, F.75252 Paris cedex 05, France
}

\begin{abstract}
:a new method for obtatning the parametric sound field is performed, which combines the parabolic approximation and the expansion of the source condition in a sum of Gaussians. This leads to a single integral easy to compute numerically. The main features of the parametric sound field are studied afterwards.
\end{abstract}

\section{INTRODUCTION}

L'émission paramétrique est un moyen d'obtenir un faisceau acoustique borné basse fréquence et directif en utilisant des transducteurs de dimensions modérées. Le principe en est le suivant : une source acoustique émet deux signaux, dits primaires, H.F. directifs. Les interactions non linéaires de ces signaux dans le fluide produisent un signal (paramétrique), dont la fréquence est la différence des fréquences primaires. Si celles-ci sont voisines, le signal-résultant sera B.F., donc peu atténué mais de directivité comparable à celle des faisceaux primaires.

Si le principe de l'émission paramétrique est connu depuis longtemps [1], le calcul effectif du champ requiert des moyens numériques coûteux $[2,3]$. De nombreux modèles ont été développés [4] afin d'obtenir des expressions simples. Un modèle est ici proposé, basé sur l'utilisation conjointe de l'approximation parabolique (I) et d'une décomposition de la condition de source en somme de gaussiennes (II). Ces deux hypothèses permettent d'obtenir une expression analytique du champ paramétrique sous une forme simple (III) aisée à calculer numériquement. Les caractéristiques essentielles du champ paramétrique sont retrouvées : directivité élevée et lobes secondaires peu marqués (IV).

\section{L'APPROXIMATION PARABOLIQUE EN ACOUSTIQUE NON LINEAIRE}

Celle-ci est basée sur une étude dimensionnelle [5] des mécanismes intervenant dans 1a propagation des faisceaux bornés non linéaires, dont les ordres de grandeur sont mesurés par différents petits paramètres : nombre de Mach $M=V / c$ (V : amplitude à la source, $c$ : célêrité du son) pour les non-linéarités, le nombre de Stokes $S=k D / c(\omega$ : fréquence de référence, $k=\omega / c$, $D$ : diffusivité du son) pour la dissipation thermovisqueuse, parametre $\varepsilon=1 /(\mathrm{ka})$ (a : rayon de la source) pour la diffraction. Dans le cadre de $1^{\prime}$ émission paramétrique, ces paramètres sont tou- 
jours três petits devant $1^{\prime}$ unité. Ceci traduit le fait que l'onde peut être considérée comme localement plane : elle ne dépend sur quelques longueurs d'onde que de la variable caractéristique $T=\omega(t-x / c)$ d'une onde plane le long de $I^{\prime}$ axe acoustique $\mathrm{Ox}$ (Fig. 1). Les divers mécanismes $\mathrm{n}^{\prime}$ agissent que sur des distances beaucoup plus grainde. En appliquant avec ces hypothèses le formalisme des échelles multiples aux équations de Navier - Stokes, on aboutit à 1 'équation dite KZK [6,7]:

$$
\frac{\partial^{2} U}{\partial \delta \partial \tau}-\frac{1}{4} \Delta_{\perp} U-\frac{1}{\Pi N} \frac{\partial^{3} U}{\partial T^{3}}=\frac{1}{N} \frac{\partial}{\partial \tau}\left(U \frac{\partial U}{\partial \tau}\right)
$$

avec $\beta$ le paramètre de non-linéarité et :

$U=u_{x} / U_{0}, \delta=2 x / k^{2}, R=\sqrt{y^{2}+z^{2}} / a, \Delta_{\perp}=\partial^{2} / \partial R^{2}+1 / R \partial / \partial R, \Gamma=\beta M / S, N=2 / \beta M(k a)^{2}$.

\section{DECOMPOSITION DE LA SOURCE EN SOMME DE GAUSSIENNES}

Dans le cas linéarisé $(\mathrm{N}=+\infty)$, une solution exacte de $I^{\prime}$ approximation parabolique est connue pour une source monochromatique et gaussienne :

$$
U(\delta, R, \tau)=\frac{A}{1-i \delta B} \exp \left(-\frac{B^{2}}{1-i \delta B}-\frac{\delta}{\Gamma N}+i \tau\right)
$$

La forme simple de cette solution suggère de rechercher des solutions plus générales de l'équation KZK linéarisée sous forme d'une somme de telles expressions en approchant la condition de source exacte par une somme de gaussiennes [8] :

$$
U(\delta=0, R, T)=U_{0}^{e x}(R) e^{i \tau} \approx U_{0}^{a p}(R) e^{i \tau} \quad \text { où } \quad U_{0}^{a p}(R)=\sum_{n=1}^{N} A_{n} \exp \left(-B_{n} R^{2}\right)
$$

Ceci peut être fait soit par optimisation [9], soit par identification des premiers termes des développements de Taylor des transformées de Hankel des conditions exactes et approchées [5]. La figure 3 illustre une telle décomposition pour une condition uniforme de type piston et pour 1,2 ou 10 gaussiennes. L'amplitude du champ proche créée par de telles sources sur l'axe est visualisée sur la figure 3 qui montre que, plus le nombre de gaussiennes retenues est élevé, plus le champ approché fournit une description précise et valable plus près de la source.

\section{CALCUL DU CHAMP PARAMETRIQUE}

Les considérations précéđentes vont nous permettre d'évaluer le champ paramétrique produit par une source bichromatique gaussienne :

$$
U(\delta=0)=U_{0}^{a p}(R)\left(e^{i \tau}+e^{i \eta \tau}\right) \quad(\eta: \text { rapport des fréquences primaires })
$$

Pour ce faire, on recherche une solution approchêe de 1 'équation KZK par développement en puissances de $1 / \mathrm{N}$. La composante $U_{-}$de fréquence différence $\eta-1$ du 
terme de second ordre se calcule par transformée de Hankel [5]:

$$
\begin{array}{cc}
U_{-}=-\frac{1}{2 N} \operatorname{Re}\left\{(\eta-1)^{2} V_{-} \exp \left(-(\eta-1)^{2} \delta / \Gamma N+i(1-\eta) \tau\right)\right\}, \\
V_{-}=i \int_{0}^{\delta} \sum_{n=1}^{N} \sum_{m=1}^{N} A_{n} A_{m}^{*} \frac{e^{-\alpha x}}{c_{n m} x+d_{n m}} \exp \left(-R^{2} \frac{a_{n m} x+d_{n m}}{c_{n m} x+d_{n m}}\right) d x \quad(\alpha=2 \eta / \Gamma N), \\
a_{n m}=-i B_{n} B_{m}^{*}(\eta-1)^{2} / \eta & b_{n m}=(\eta-1)\left(B_{n}+B_{m}^{*}\right) \\
c_{n m}=\delta(\eta-1) B_{n} B_{m}^{*} / \eta-i \eta B_{n}-i B_{m}^{*} / \eta & d_{n m}=\eta-1+i \delta\left(\left(B_{n}+B_{m}^{*}\right) .\right.
\end{array}
$$

Pour un point d'observation situé sur l'axe ( $\mathrm{R}=0), 1$ 'intégration numérique de (5) requiert environ 0,5s de temps CPU sur Apollo 4000 (1'intégrale s'explicite sous forme d'exponentielles intégrales) pour $\mathrm{N}=10$ gaussiennes, et $14 \mathrm{~s}$ hors de 1 'axe.

\section{ANALYSE DU CHAMP PARAMETRIQUE}

Nous noterons ici seulement les caractéristiques les plus marquantes du champ paramétrique en fonction de 1 'atténuation $\alpha$ et du rapport $\eta$ des fréquences primaires. En champ proche il est croissant jusqu'à environ 0,5 fois la distance de Rayleigh (si l'atténuation est faible) (Fig. 4), puis décrô̂t lentement en ln $\delta / \delta$ jusqu'ầ la fin de la zone d'interaction déterminée par la distance 2/ $\alpha$ (au-delà les champs primaires sont totalement atténués). Les diagrammes de directivité montrent des lobes secondaires peu marqués et de niveau très bas (-50dB à la distance de Rayleigh, Fig. 5). En champ lointain, l'angle de demi-ouverture passe par un minimum situé grossièrement à la distance $2 / \alpha$ (Fig. 6) et d'autant plus marqué que la fréquence paramétrique est basse.

\section{CONCLUSION}

La simplicité des résultats permet d'envisager la généralisation à des situations plus complexes (sources focalisées ou rectangulaires, interaction avec une interface). Il est également possible d'étudier l'amélioration du renđement de l'émission paramétrique par optimisation, soit de la condition de source (c'est-à-dire des paramètres $A$ et $B$ ), soit du signal temporel (signal multifréquentiel).

[1] P.J. Westervelt, J. Acoust. Soc. Am., 35, 535-537, 1963

[2] P. Alais et P.Y. Hennion, Acustica, 43, 1-11, 1979

[3] THOMSON SINTRA ASM, contrat DRET 85/40501, rapport final, 1987

[4] B.K. Novikov, O.V. Rudenko et V.I. Timoshenko, "Nonlinear Underwater Acoustics", American Institute of Physics, INC, New York, 1987

[5] F. Coulouvrat, Thèse de Doctorat de I'Université Pierre et Marie Curie

[6] E.A. Zabolotskaya et R.V. Khokhlov, Sov. Phys. Acoust., 15, 35-40, 1969

[7] V.P. Kuznetsov, Sov. Phys. Acoust., 16, 467-470, 1970

[8] R. Burvingt, Revue du CETHEDEC, 48, 195-218, 1976

[9] J.J. Wen et M.A. Breazeale, J. Acoust. Soc. Am. , 83, 1752-1756, 1988 
Rgure 1 ; postion du probllme
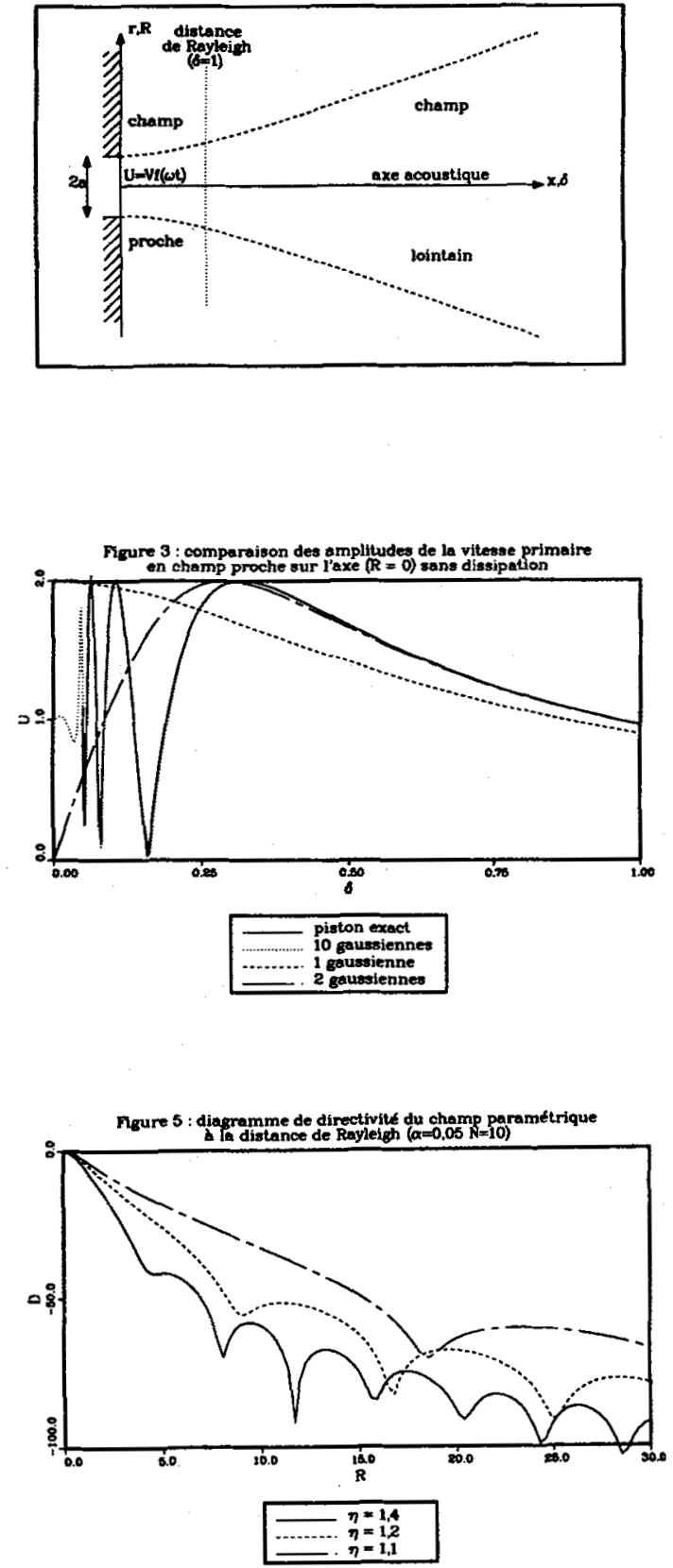
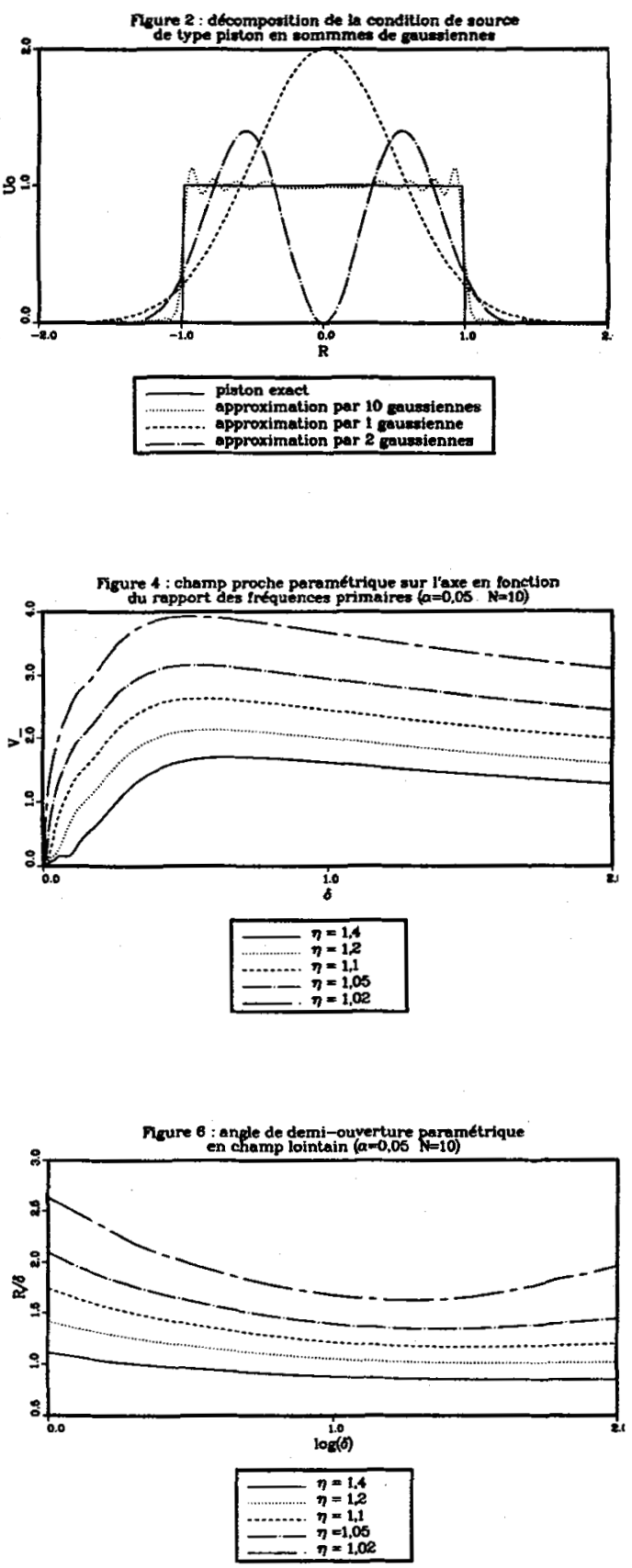